\title{
¿Es POSIble APRENDER INDUCTIVAMENTE de LA EXPERIENCIA?
}

\author{
Dayron Alberto Arboleda Quintero ${ }^{1}$ \\ Margarita Emilia Patiño ${ }^{2}$
}

\section{Resumen}

Con el surgimiento del cálculo de probabilidades como una nueva metodología de la cuantificación del riesgo, nace la discusión respecto a la viabilidad de la probabilidad inductiva, o si la estadística clásica utiliza la metodología inductiva o deductiva para la obtención de sus conclusiones, base del método científico; polémica que se agudiza con el afianzamiento de la estadística bayesiana, que al parecer es la respuesta más prometedora a favor del aprendizaje inductivo y que es defendida por los autores a lo largo de este artículo. Son muchos los filósofos que niegan que sea posible aprender inductivamente desde la experiencia. Las objeciones más conocidas al método inductivo provienen de David Hume y posteriormente de Karl Popper, que, entre otros, rechazan toda posibilidad de una lógica inductiva.

\section{Palabras clave}

Filósofos, Aprendizaje inductivo, Método, Estadística bayesiana, Cálculo de probabilidades, Aprendizaje deductivo.

1 Ingeniero Civil. Especialista en gestión energética industrial y candidato a doctor UPV / EHU. Integrante del grupo de investigación Davinci del ITM.

2 Ingeniera Química, UdeA. Especialista en docencia universitaria. Posgrado en Didáctica de las Ciencias con énfasis en matemáticas y física. 


\section{Abstract}

With the birth of probability calculus as a new risk quantification methodology, it also appears the debate with respect to the viability of inductive probability or to the fact that classic statistics uses inductive or deductive methods to draw its conclusions which is the basis of the scientific method. Such discussion becomes more intense with the strengthening of Bayesian statistics that seems to be the most promissory answer in favor of inductive learning and which is supported here by the authors of this article. There are many philosophers that reject the possibility to learn inductively from the experience. The most famous objections in this sense come from David Hume and, then, from Karl Popper who, among others, resist any possibility of an inductive logic.

\section{Key words}

Philosophers, Inductive Learning, method, Bayesian Statistics, Probability Calculus, Deductive Learning. 


\section{INTRODUCCIÓN}

En el proceso de la construcción del conocimiento se han usado generalmente dos tipos de razonamiento: el deductivo y el inductivo ${ }^{3}$. El hecho de que la inducción sea ampliativa (es decir parte de lo particular a lo general), ha seducido a gran cantidad de filósofos y científicos que pretenden predecir lo que va a ocurrir en el futuro, basándose en las evidencias empíricas del pasado. Dado que la inducción parte de premisas particulares de un subconjunto del conjunto de los naturales, a leyes generales que se cumple para todo el conjunto de los naturales, se genera pesimismo en cuanto a la dificultad de su entendimiento, justificación o validez, lo que ha desembocado en interminables debates entre filósofos y científicos en los últimos siglos.

Para abordar el papel de la inducción en la formulación de modelos y así realizar una propuesta de solución sobre los principales puntos en discordia, se han fijado tres objetivos relacionados con cuestionamientos básicos acerca de la metodología inductiva, respecto a los cuales se defienden unas tesis muy concretas y específicas.

Los objetivos fijados relacionados con la metodología inductiva son:

- Dilucidar el papel del método inductivo para la generación de nuevo conocimiento, de tal forma que se develen las virtudes y falencias de esta metodología desde una perspectiva pragmática.

- Justificar el uso de la inferencia inductiva como una de las metodologías apropiadas para aprender de la experiencia, dentro de un contexto concreto y específico.

3 En el razonamiento deductivo la conclusión está contenida en las premisas, por lo que, si partimos de un conocimiento verdadero, obtenemos otro conocimiento también cierto. Por el contrario, en el método inductivo la conclusión es más amplia que la información contenida en las premisas; siendo por lo tanto posible que la conclusión sea falsa, aunque las premisas sean verdaderas. (DÍAZ 2007, pág. 39). 
- Comprender el alcance y las limitaciones de la estadística inferencial clásica y profundizar en los métodos bayesianos, como una posible alternativa en la solución de problemas, donde el conocimiento subjetivo del investigador tiene un alto grado de ponderación en la toma de decisiones para la solución de éstos.

En éste artículo se abordan tres cuestionamientos relacionados con el método inductivo: ¿Es la estadística clásica inductiva o deductiva?, para dar respuesta se defiende la siguiente tesis:

La estadística clásica es deductiva desde la planeación de su estrategia de muestreo o diseño experimental, inductiva desde la inferencia de sus resultados y deductiva en la aplicación de sus resultados, donde también se deja abierta la posibilidad de obtener modelos inductivamente.

¿Es posible aprender inductivamente de la experiencia? Para dar respuesta se defiende la siguiente tesis:

Si es posible aprender inductivamente de la experiencia usando técnicas estadísticas clásicas y/o bayesianas, siempre y cuando se considere el contexto, como un factor determinante para la validez y fiabilidad de estas metodologías ${ }^{4}$.

¿Cuál es la metodología estadística más apropiada para la formulación de modelos estadísticos para dar respuesta, se defiende la siguiente tesis:

Ante las limitaciones de los modelos estadísticos clásicos ${ }^{5}$, el bayesianismo surge como una alternativa para la toma de deci-

4 Como se verá posteriormente, hay autores que no conciben un híbrido de estadística clásica y bayesiana; pero yo coloco clásica y/o bayesiana, porque en la actualidad las dos metodologías vienen siendo usadas paralelamente sin suponer un dramático cambio de paradigma del frecuentismo por el bayesianismo. De acuerdo al problema a resolver se adapta mejor una metodología que la otra. Por ejemplo, en epidemiología el bayesianismo es cada vez más utilizado, lo mismo que en el estudio de fenómenos climáticos donde la estadística clásica ha mostrado limitaciones y falencias. Recordemos que a pesar de los revolucionarios cambios con la física relativista, los ingenieros todavía usan principios de la mecánica Newtoniana.

5 La estadística clásica o frecuencialista (frecuentista) es la que utiliza los métodos de máxima verosimilitud de Ronald Fisher y los intervalos de confianza de Neyman 
siones, considerando el conocimiento sustantivo del investigador para la solución de un problema específico.

Antes de tratar de dilucidar los tres cuestionamientos mencionados, se abordan brevemente los esquemas clásicos de inducción y los problemas asociados a esta metodología, desde una perspectiva moderna.

La cuestión acerca de si es posible aprender inductivamente de la experiencia, ha preocupado a los filósofos desde tiempos remotos. La respuesta de Aristóteles según la cual la inducción enumerativa garantiza la inferencia de verdades empíricas universales a partir de un número finito de observaciones había sido aceptada ampliamente, hasta que David Hume y posteriormente Popper argumentaron la ilegitimidad lógica de la inferencia ampliativa y conservadora de la verdad (Rivadulla 1993, pág. 3).

La atribución de valores de probabilidad a las conclusiones obtenidas de observaciones metódicas transformó la cuestión de la validez de la inducción en la de la posibilidad de la probabilidad inductiva. Las raíces de este cambio hay que buscarlas, empero, en la historia del cálculo de probabilidades, no en la de la filosofía.

En efecto, años atrás, en la segunda mitad del siglo XVIII, se había publicado un trabajo del clérigo inglés Thomas Bayes, en el que por primera vez permitió un tratamiento matemático de la inferencia inductiva (referencias).

Ya en el siglo pasado proliferaron tres grandes familias de la inferencia probabilista: la teoría de la estimación y contraste de hipótesis (estadística frecuentista) de Fisher, Neyman, Pearson (crearon metodologías basadas en la concepción frecuencialista de la probabilidad), dando lugar al método conocido como prueba

y Pearson. Se diferencia de la estadística bayesiana (Bruno De Finetti, Savage y otros) en que no utiliza probabilidades a priori como sí lo hace el bayesianismo y por lo tanto, no tiene en cuenta el conocimiento acumulado que tiene el investigador acerca del problema en cuestión. Además la estadística clásica no puede asignarle probabilidades a una hipótesis (como sí lo hace el bayesianismo) y simplemente la rechaza o se reserva el juicio, para no comprometerse aceptándola. 
de significación, el que tiene en cuenta un índice para medir la fuerza de la evidencia, denominado valor $p$, $y$ la hipótesis, llamado prueba de hipótesis, $\mathrm{PH}$.

Una segunda familia es la inferencia bayesiana (Jeffreys, De Finetti, Savage, Lindley). En la teoría de la decisión (Wald), la tercera familia, el problema fundamental radica en cómo cuantificar la incertidumbre asociada a los fenómenos aleatorios, que constituyen el dominio de aplicación de la inferencia estadística (Rivadulla 1991, pág. 11).

Antes de abordar los tres cuestionamientos básicos planteados, es importante acotar la discusión en los siguientes aspectos:

- La inducción enumerativa ha sido la más cuestionada, pero como señala Norton (2003, pág. 4), la ciencia siempre ha usado inducción enumerativa y esto no es posible que cambie: creemos que todos los electrones tienen una carga de $1.6 * 10^{-19}$ coulumbios, porque todos los electrones medidos hasta ahora llevan esa carga. Abundan los contraejemplos que buscan invalidar la generalización inductiva, que no consideran el hecho de que toda inducción conlleva riesgo. No se puede rechazar una medicina que ha curado a 100 personas porque a la siguiente no la curó. Por eso, es importante replantear los viejos problemas de la inducción hacia problemas más modernos de corte pragmático ${ }^{6}$.

- También se considera importante despojar la discusión de ese carácter bipolar ingenuo, donde no se permite que en la ciencia convivan inducción/deducción, continuidad/discontinuidad, teo-

6 Hay un problema que necesita una pronta solución y está relacionado con la efectividad de las pruebas estadísticas clásicas en un contexto no uniforme. En los últimos años se ha complicado la predicción del clima y sólo se puede hacer con tres días de antelación, lo que no ocurría veinte años atrás y lo cual puede ser debido al trastorno medio ambiental inducido por el hombre. Es como una premonición Baiconiana del concepto de uniformidad de la naturaleza. Las predicciones del comportamiento de las bolsas de valores vienen perdiendo credibilidad e incluso se está recurriendo a la experiencia acumulada por los expertos a través de varios años. 
ría/experimentación. Así como la teoría de conjuntos cantoriana no fue suficiente para resolver ciertos problemas y le tuvo que abrir paso a una nueva lógica ${ }^{7}$ con una gama de posibilidades más amplia, donde no se habla de pertenece/no pertenece o verdadero/falso, la filosofía de la ciencia debe cerrar por completo los debates nocivos para el avance de ésta. Ya no tiene sentido hablar de verdadero o falso, ni discutir si el progreso científico es continuo o funciona por la ruptura de paradigmas. En una postura pragmática se deben usar los modelos y las teorías mientras sean útiles y no permitir, por ejemplo, que mueran millones de personas de cáncer esperando el tratamiento ciento por ciento efectivo. Todos los modelos son inexactos, aproximados y la estadística sea clásica o bayesiana, va a estar siempre sujeta a errores. La inducción perfecta es una utopía.

\section{2. ¿Es la eStadística CLÁSiCa InduCtiva O DEDUCtiva?}

Con la contribución del cálculo de probabilidades al análisis del riesgo, la controversia acerca de la validez del método inductivo se transforma en la posibilidad de una probabilidad inductiva y se vislumbran argumentos a favor de la factibilidad de aprender inductivamente de la experiencia; sobre todo, a medida que la estadística desarrolla novedosos métodos para resolver problemas de tipo práctico ${ }^{8}$. Aunque el enemigo más recalcitrante de la inducción,

7 La teoría de conjuntos de George Cantor es de naturaleza bipolar (bueno/malo, prendido/apagado, bonito/feo, verdadero/falso... etc.) donde un elemento pertenece o no pertenece a un conjunto. Esto trae nefastas limitaciones en su aplicación y por ejemplo, no hay un conjunto donde ubicar a una droga $80 \%$ efectiva. En la lógica difusa, un elemento puede pertenecer parcialmente a un conjunto, lo que posibilita grandes aplicaciones a nivel tecnológico. Mientras esta lógica permite inducciones probabilistas, la teoría cantoriana no encaja en este esquema.

8 Ronald Fisher se interesó por la estadística al estudiar errores en las observaciones astronómicas. En 1919 empezó a investigar en biología en problemas de genética y teoría de la evolución. Fisher fue el descubridor de los test de significación estadística, que perfeccionaron Neyman y Pearson al introducir la hipótesis alternativa y los errores tipo I y tipo II. 
Popper, argumenta que no existe fundamento lógico ni psicológico para la probabilidad inductiva (Banegas 2000, pág. 328); se cree que el debate se inclina un poco a favor de los inductivistas y los detractores de la inducción reorientan sus argumentos, hacia el carácter deductivo de la estadística clásica. En la actualidad es más cómodo para un filósofo anti-inductivista, argumentar que la estadística es deductiva, que empeñarse en desconocer que se puede aprender inductivamente de la experiencia, porque son innumerables las evidencias pragmáticas que confirman esta posibilidad ${ }^{9}$. La inferencia inductiva nos posibilita a través de la experiencia ampliar nuestro conocimiento empírico (Vickers 2006, pág. 5).

Aunque Ronald Fisher, el padre de la estadística moderna, postulaba su carácter inductivo de la significación estadística y la estimación de parámetros, algunos autores argumentan que se trata de un razonamiento deductivo: se trata de un razonamiento a partir de una distribución conocida (la del estadístico en todas las posibles muestras de la población) hacia uno de sus valores (la muestra que tomamos para el experimento). La distribución en el muestreo es conocida y a partir de ella se calcula la probabilidad del valor particular obtenido, lo cual es un razonamiento deductivo (Díaz 2007, pág. 42). (Rivadulla 1991, pág. 144, 145) analiza críticamente el método fisheriano de máxima likelihood ${ }^{10}$

9 En el apartado 3, mostramos con ejemplos muy concretos de tipo pragmático, cómo sí es posible aprender inductivamente de la experiencia, sin necesidad de recurrir siquiera a la inducción probabilista. Los modelos de regresión se obtienen matemáticamente por deducción, pero son inductivos a nivel pragmático, en el sentido que puedo formular un modelo general a partir de unos pocos datos experimentales. También en esta sección se abordarán problemas reales donde la estadística clásica ha mostrado grandes virtudes para la toma de decisiones al futuro, basándonos en la experiencia del pasado.

10 Uno de los métodos más usados para obtener una estimación puntual de un parámetro poblacional, es el método de máxima verosimilitud: un conjunto de observaciones $x_{1}, x_{2} \ldots x_{n}$ constituye una muestra aleatoria de tamaño $n$ de la población infinita $f(x)$ si:

1. Cada $x_{i}$ es un valor de una variable aleatoria cuya distribución está dada por $f(x)$. 
y remata afirmando: me parece importante subrayar que, contra la opinión defendida por Fisher, ni el método de máxima likelihood, ni el argumento fiducial constituyen procedimientos capaces de garantizar el aprendizaje inductivo a partir de la experiencia (Rivadulla 1991, pág 146).

Neyman y Pearson, junto con Fisher los forjadores de la estadística moderna, defienden el carácter deductivo de ésta y para los test de hipótesis introducen el concepto de hipótesis alternativa y hablan de dos tipos de error. En esta metodología las probabilidades de error son iniciales y no finales. Por tanto, no es posible calcular inductivamente la probabilidad de la hipótesis a partir de los datos, ya que este procedimiento es deductivo (Díaz 2007, pág. 44).

No se niega el carácter deductivo de la estadística frecuentista, e incluso paradójicamente el teorema de Bayes se obtiene deductivamente a partir de los axiomas básicos de probabilidad ${ }^{11}$; lo que se pretende mostrar es que la estadística clásica es deductiva en su desarrollo e inductiva en su aplicación. Es deductiva en su desarrollo, en el sentido de que sus teoremas generalmente se obtienen matemáticamente a partir de otros axiomas ya aceptados, similar a como ocurre con la geometría euclidiana. Sin embargo, hay modelos muy importantes de la estadística que se

2. Éstas $n$ variables aleatorias son independientes. Supóngase que $x$ es una v.a con distribución de probabilidad $f(x, \theta)$, donde $\theta$ es un parámetro desconocido. Sean $x_{1}, x_{2} \ldots x_{n}$ los valores observados en una muestra aleatoria de tamaño $n$. Entonces la función de probabilidad de la muestra es $L(\theta)=f\left(x_{1}: \theta\right) \cdot f\left(x_{2}: \theta\right) \ldots f\left(x_{n}: \theta\right)$ . La función de probabilidad sólo depende del parámetro desconocido $\theta$. El estimador de máxima verosimilitud de $\theta$, es el valor de $\theta$ que maximiza la función de probabilidad $L(\theta)$.

11 Si los eventos $B_{l}, B_{2}, \ldots . K_{k}$, de un espacio muestral $\mathrm{S}$, constituyen una partición $\left(\sum p\left(B_{i}\right)=1\right.$ y $\left.p\left(B_{i} \cap B_{j}\right)=0\right) i \neq, j$. Para cualquier evento $A \in S$ se cumple: $p(A)=\sum_{i=1}^{k} B_{i} \cap A=\sum_{i=1}^{k} p\left(B_{i}\right) p\left(A / B_{i}\right)$ por la definición de probabilidad condicional $p\left(B_{r} / A\right)=\frac{p(B r \cap A)}{\sum_{i=1}^{k} B_{i} \cap A}=\frac{p\left(B_{r}\right) p\left(A / B_{r}\right)}{p(A)}$ 
obtienen inductivamente a partir de casos particulares; lo cual se pueden sustentar detalladamente con los modelos binomial, hipergeométrico o con el teorema central del límite. Es decir, que planteamos un doble carácter inductivo/deductivo de la estadística sea frecuentista o bayesiana.

La estadística es netamente inductiva en su aplicación, porque siempre inferimos de la muestra a la población, del pasado al futuro, de lo conocido a lo desconocido. Acorde a todos los esquemas inductivos, los métodos estadísticos nunca pueden hacer afirmaciones cien por ciento certeras, siempre llevan asociado una incertidumbre de llegar a conclusiones falsas, aún partiendo de premisas (resultados experimentales) ciertas.

Lo que hace útil a la estadística a nivel pragmático es, precisamente, ese carácter inductivo que nos permite trabajar con muestras y no con la población completa, lo cual arrojaría resultados certeros como en la metodología deductiva, pero a un costo muy alto $^{12}$.

Una hipótesis estadística es una aseveración o conjetura acerca de los parámetros de una o varias poblaciones. La verdad o falsedad de una hipótesis estadística siempre estará sujeta a los resultados de una muestra aleatoria de la población. Ejemplos de interés para el lector que defienden la idea del inductivismo por medio del uso de las probabilidades, se pueden ver en Walpole/Myers, 2007 págs. $154,245,246$ y 321.

12 Pensemos si tendría sentido encuestar a todos los posibles votantes de un país, para vaticinar quién será el próximo presidente. Hay ensayos de tipo destructivo, por ejemplo, para cuantificar la resistencia de cierto material, donde mientras más grande sea la muestra, más confiables son los resultados pero a su vez más costoso el experimento. El investigador busca un punto de equilibrio entre estos dos factores (confiabilidad/costo). El gran objetivo de la estadística consiste en estimar a partir de una muestra un parámetro poblacional y someter a test de hipótesis estos parámetros. 


\section{3. ¿ES POSIBLE APRENDER INDUCTIVAMENTE DE LA EXPERIENCIA?}

Como se mencionó anteriormente, la cuestión acerca de si es posible aprender inductivamente de la experiencia, ha preocupado a los filósofos desde tiempos remotos. La respuesta de Aristóteles según la cual la inducción enumerativa garantiza la inferencia de verdades empíricas universales a partir de un número finito de observaciones había sido aceptada ampliamente; hasta que David Hume y posteriormente Popper argumentan la ilegitimidad lógica de la inferencia ampliativa y conservadora de la verdad (Rivadulla 1993, pág. 3). Posteriormente, por la inclusión del cálculo de probabilidades y del teorema de Bayes, que permite un tratamiento matemático de la inferencia inductiva, los matemáticos entran activamente en el debate y se reorienta la cuestión de la validez de la inducción hacia la posibilidad de la probabilidad inductiva, la cual también es rechazada por Popper ${ }^{13}$. Son muy pocos los filósofos o matemáticos que asumen esta posición tan radical de Popper de negar por completo todo tipo de inducción y algunos asumen una posición más mesurada, negando el carácter inductivo de la inferencia estadística, sin cuestionar sus grandes virtudes teóricas y pragmáticas ${ }^{14}$.

En la sección anterior, presentamos argumentos a favor del doble carácter inductivo/deductivo de la estadística clásica (fre-

13 Popper argumenta que no existe fundamento lógico ni psicológico para la probabilidad inductiva y choca frontalmente con la concepción defendida por Reinchenbach acerca de la decidibilidad inductivo-probabilística de las proposiciones generales de la ciencia. (Rivadulla 1991, pág. 15). Popper aboga por la imposibilidad de alcanzar inductivamente conocimiento cierto o incluso probable a partir de los siempre finitos datos de la experiencia; pues siempre podría haber una nueva observación que refutara la proposición (Banegas 2000, pág. 318).

14 Andrés Rivadulla (1991, 1993, 1996) argumenta que la estadística clásica es deductiva y que por lo tanto no es posible aprender inductivamente de la experiencia, afirma que desde un punto de vista lógico-subjetivista, la posibilidad de la probabilidad inductiva es indiscutible (1991, pág. 208). (Banegas, Rodríguez, del Rey, 2000) se adhieren a la posición de Popper de que no es posible aprender inductivamente de la experiencia y defienden el carácter deductivo de la estadística clásica, al tiempo que lanzan fuertes críticas al bayesianismo. 
cuentista) y en la sección próxima, confrontamos las posibilidades de la estadística bayesiana con respecto a la frecuentista. No utilizamos argumentos de tipo lógico, sino más bien concretos y de tipo pragmático; pero no desestimamos y nos parece muy importante profundizar en algunos enfoques a favor de la probabilidad inductiva, que no es posible abordar en el artículo: controversia Popper-Carnap y las contribuciones de Jaakko Hintikka al perfeccionamiento de la teoría de la generalización inductiva ${ }^{15}$.

Para defender la tesis de que sí es posible aprender inductivamente da la experiencia, usando técnicas estadísticas (llámese clásicas o bayesianas), aceptamos de entrada que hay que tener en cuenta el contexto, como un factor determinante para la validez y fiabilidad de estas metodologías. Implícitamente, también estamos dando por hecho que la estadística admite el razonamiento inductivo, el cual al despojarlo de pretensiones de universalidad tiene dos características básicas muy marcadas: normalmente el razonamiento inductivo va de lo particular a lo general, y desde el sentido estadístico nunca se tiene absoluta certeza acerca de la veracidad de una hipótesis.

Tanto la estadística clásica como la bayesiana presentan estas dos características básicas de la inducción:

- Van de lo particular a lo general, porque a partir de unos pocos casos particulares (los datos u observaciones) obtengo un modelo general (pero no universal).

- Tanto en la estadística clásica como en la bayesiana se habla de niveles de confianza y, por lo tanto, de márgenes de error

15 (Rivadulla 1991), (Aisa 1994), abordan adecuadamente el problema de la inducción en forma cronológica Aristóteles-Bacon-Hume-Popper y se detienen a discutir profundamente los enfoques probabilistas y las múltiples posibilidades que le abren a la inferencia inductiva. Desarrollamos la discusión no desde el punto de vista lógico-inductivo, sino cuestionando el carácter de la estadística clásica, aceptando de entrada que es una herramienta para aprender de los experimentos. 
que aparecen explícitos en ambas metodologías. Todo modelo estadístico es inexacto y aproximado.

Hay ejemplos, como los que se referenciarán a continuación, que defienden nuestra tesis de que sí es posible aprender inductivamente de la experiencia. Se pueden identificar claramente estas dos características del método inductivo y el hecho de que las inferencias son válidas, sólo dentro de un contexto específico (universo reducido/rango limitado).

A continuación, hacemos una breve sustentación acerca de la importancia del contexto para la validez de una proposición, modelo o hipótesis:

Cuando una persona no tiene ningún conocimiento teórico de las leyes que gobiernan la caída de los cuerpos, observa que si lanza hacia arriba varios objetos, todos caen al suelo. Por inferencia inductiva, interioriza la creencia de que si se lanza de cien metros de altura, va a perder la vida. A pesar de que teóricamente (deductivamente) no entiende la razón de este hecho, su inferencia es cierta y seguirá siendo cierta en cualquier punto de la Tierra. Pero, si se traslada nuestro escenario a otro planeta (contexto) como la Luna, ¿seguirán siendo válidas sus inferencias? Por supuesto que no.

¿Te atreverías a vaticinar el comportamiento de la bolsa de valores en Inglaterra o más bien en Etiopía?

$¿$ Es igual de fiable y sencillo vaticinar el estado del clima para el próximo abril en la contaminada ciudad de México, que en la selva amazónica virgen?

Considerando que la inferencia estadística clásica no facilita la predicción de resultados futuros basándose en la evidencia empírica del pasado, es lógico pensar que funciona mejor en la medida que las condiciones no cambien desmesuradamente en el intervalo de tiempo en consideración. Es decir, condiciones de homogeneidad y uniformidad, que cada vez son menos factibles en la naturaleza y en la sociedad. Hace tres décadas el clima mundial era más uniforme que en la actualidad y por lo tanto su predicción era más confiable. En la actualidad sólo se puede vaticinar con tres días 
de antelación. Las inferencias de tipo social y político también son afectadas directamente por el contexto ${ }^{16}$.

- Una gran cantidad de modelos usados en la ciencia son válidos bajo ciertas limitantes: presiones bajas, temperatura constante, demanda uniforme... etc. Es decir, en un contexto específico. En los modelos de regresión, la validez está sujeta a las condiciones en que se realiza el experimento y solo son fiables en el rango experimental en que se toman los datos.

Se está proponiendo la validez de la inducción, pero dentro de un contexto específico; lo cual tiene afinidades con la teoría de Norton (2003). Este es reiterativo en que las inducciones son siempre autorizadas por postulados materiales que sólo prevalecen en dominios locales (2003, pág. 28). Norton no sólo vislumbra una visión pragmática de la inducción, sino que refleja claramente su renuncia a la universidad y a la generalidad.

Se considera que no es necesario renunciar a la generalidad, siempre y cuando se acote el contexto en el cual es válido el modelo y se enuncien las idealizaciones y las cláusulas ceteris paribus a las cuales está sujeto. Por ejemplo, los modelos de regresión son obtenidos deductivamente, pero en su aplicación son inductivos, porque se obtienen a partir de casos particulares, que limitan el rango en el cual es válido el modelo.

\subsection{Argumentos a favor de la obtención de conocimiento inductivamente}

A continuación se presentan tres casos concretos, donde se genera nuevo conocimiento inductivamente o se obtienen argumentos para tomar una decisión respecto a una situación específica.

16 Gran cantidad de factores pueden afectar la obtención de resultados óptimos por inferencia estadística: intereses de tipo particular o político sesgan los resultados de los sondeos de opinión, relacionados con las preferencias de los ciudadanos por un producto comercial o por un candidato al gobierno. Aquí es muy importante el contexto, porque hay países que ejercen un control más serio y riguroso a estos sondeos. 
En Walpole/Myers 2007, pág. 148, en el planteamiento de un problema específico de cálculo de probabilidades se afirma que hay impurezas en $30 \%$ del total de pozos de agua potable de cierta comunidad rural. Para obtener algún conocimiento real del problema, se determina que debe realizarse algún tipo de prueba. Es muy costoso probar todos los pozos del área, por lo que se eligen diez aleatoriamente para la prueba. Después del experimento, resulta que seis de los pozos chequeados contienen impurezas. ¿Qué implica esto respecto a la conjetura que se tenía inicialmente; que el 30\% del total tienen impurezas?

En su solución, ver (Walpole/Myers 2007, pág. 148), realmente resultan 6 pozos con impurezas, es muy poco probable (4.73\%) que esto hubiera sucedido, en caso de ser cierta la afirmación de que el 30\% de todos los pozos están contaminados.

El resultado de este problema obtenido empíricamente por inducción, me brinda un conocimiento más real de la verdadera dimensión del fenómeno y sugiere que el problema de la contaminación de los pozos es mucho más severo.

Los test de hipótesis estadísticas ${ }^{17}$ tienen afinidades con el planteamiento anterior y nos brindan argumentos para dudar de una afirmación respecto a un parámetro poblacional.

Otros ejemplos muestran cómo a partir de varios casos particulares (medidas empíricas) se puede obtener un modelo general (válido dentro del rango experimental), que a su vez me permite realizar inferencias para valores desconocidos de la variable independiente. Si bien es cierto que los modelos de regresión se obtienen deductivamente ${ }^{18}$, en su aplicación son de carácter

17 Aunque los métodos estadísticos para probar hipótesis han variado respecto a su concepción inicial, la filosofía en el fondo es la misma: se analiza la posibilidad de que la hipótesis sea cierta, de acuerdo con unos resultados empíricos obtenidos. Una discrepancia muy fuerte entre el resultado obtenido y el esperado, me brinda argumentos para tomar la decisión de rechazar la hipótesis.

18 Para obtener el modelo de regresión lineal $y=a+b x$, se usa el método matemático conocido como de mínimos cuadrados, el cual minimiza los errores en la estimación de la variable dependiente $y$. La ecuación de la recta es $y=a+b x$ y los valores de $y$ correspondientes a los datos experimentales $\left(X_{1}, Y_{1}\right),\left(X_{2}, Y_{2}\right)-\left(X_{n}, Y_{n}\right)$ son: 
inductivo porque van de lo particular a lo general (de los datos al modelo) y siempre traen asociado una incertidumbre ya que no se ajustan exactamente a los datos.

\subsection{Determinación de variables físicas a partir de medidas experimentales}

Walpole/Myer 2007, pág. 428, muestra cómo a partir de un modelo conocido, se puede estimar el valor de una variable física, a partir de unos cuantos casos particulares. En este problema se puede deducir que el modelo que se obtiene es de la forma $y=m x+b$, donde a través de mínimos cuadrados se llega a los coeficientes de regresión: $b=14.7590, m=-2.6535, \gamma=2.6535$.

Como es del caso en los modelos, el valor obtenido no coincide exactamente con el valor real, pero es de gran utilidad a nivel práctico y se puede mejorar su exactitud, depurando los datos experimentales ${ }^{19}$.

\section{4. ¿CUÁl eS la metOdología estadística más aPROPIADA: BAYESIANISMO-FRECUENTISMO?}

El primer tratamiento matemático de la inferencia inductiva lo da el teorema de Bayes:

$$
P\left(A_{j} \mid B\right)=\frac{p\left(B \mid A_{j}\right) P\left(A_{j}\right)}{\sum_{i=1}^{K} p\left(B \mid A_{i}\right) P\left(A_{i}\right)} ; j=1, \ldots ., k
$$

$a+b X_{1}, a+b X_{2}, \ldots a+b X_{n}$. Diferenciando parcialmente la función, se obtienen los coeficientes de regresión resolviendo el sistema:

$n a+b \Sigma X=\Sigma Y$

$a \Sigma X+b \Sigma X^{2}=\Sigma X Y$

19 Existen técnicas para mejorar los valores obtenidos de los coeficientes de regresión $a$ y $b$, que consisten en eliminar los puntos experimentales que queden por fuera de la región limitada por las rectas $y=a+b x, Y=a_{\max }+b_{\max } X, Y=a_{\min }+b_{\min } X$. En el texto experimentación (1991) Baird D.C aparece explicado claramente el procedimiento para hallar los $a, b$ máximos y mínimos. 
Éste nos permite calcular las probabilidades finales, a partir del conocimiento de las probabilidades iniciales ${ }^{20} \mathrm{y}$ de los datos obtenidos experimentalmente (Días 2007, pág. 45), (Rivadilla 1991, pág. 126).

En el teorema de Bayes se trabaja de manera inductiva, ya que puede aplicarse sucesivamente de un experimento a otro, tomando los resultados del último, como probabilidades a priori iniciales, de las probabilidades finales del experimento anterior (Días 2007, pág. 45). Aunque este teorema se deduce racionalmente de los axiomas de probabilidad y de la definición de probabilidad (Wisniewski 2002, pág. 80) ha sido objeto de múltiples controversias y el punto en que más se centran éstas es en lo relacionado con la aceptación de las probabilidades a priori.

Considerando que todo cambio de paradigma científico es traumático y complicado de afianzar ${ }^{21}$, no se trata en esta sección de magnificar el bayesianismo sobre la estadística clásica, sino más bien de dilucidar las virtudes o falencias de cada metodología desde un punto de vista pragmático. Es importante entonces, empezar analizando las grandes diferencias entre el bayesianismo y frecuentismo.

Desde que se inicio al cálculo de probabilidades hasta 1950, se creía que la probabilidad de un suceso era una propiedad objetiva inherente al suceso en sí. Esta probabilidad se podía determinar de un modo objetivo de acuerdo al número de veces que ocurre el suceso en un número muy grande de repeticiones del experimento

20 (Wisniewski 2002, pág. 80) muestra la esencia del teorema de Bayes

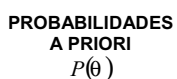

\begin{tabular}{|c|}
$\begin{array}{c}\text { PROBABILIDAD DE } \\
\text { LA EVIDENCIA } \\
\text { EMPIRICA } P(X / \theta)\end{array}$
\end{tabular}$\rightarrow$\begin{tabular}{c}
$\begin{array}{c}\text { PROBABILIDAD A } \\
\text { POSTERIORI } \\
P(X / \theta)\end{array}$ \\
\hline
\end{tabular}

21 Pensemos en las grandes repercusiones a nivel científico y social, que causó el cambio de paradigma de la física aristotélica que había sido aceptada durante 2000 años, hacia la física galileana y posteriormente mecánica newtoniana. Aceptar el bayesianismo para un experto en estadística, sería como reconocer que lo que ha hecho está mal o pudo haberlo hecho mejor. 
(probabilidad como frecuencia relativa). La estadística clásica o frecuentista pertenece a este enfoque (Rivadulla 1991, pág. 13). A partir de 1950 nace la inferencia bayesiania, que alberga la posibilidad de una probabilidad subjetiva, relacionada con nuestro grado de creencia en la ocurrencia de un evento sujeto a incertidumbres.

Un ejemplo de probabilidad frecuentista es el de un basquetbolista al que se le calcula la razón entre los tiros libres que lanza y los que acierta (supóngase que tiene una efectividad del 80\%). También se podría estimar la probabilidad como el cociente entre los casos favorables y los casos posibles de un experimento: la probabilidad de que un dado no sesgado muestre un tres es de $\frac{1}{6}$.

Hay gran cantidad de circunstancias, en donde debido a las muchas posibilidades de controlar los errores, ya sea porque son fenómenos caóticos o multicausales, no se pueden aplicar los enfoques de probabilidad anteriores y muchas personas podrían hacer diferentes estimaciones al respecto ${ }^{22}:$ ¿Cuál es la probabilidad de que llueva mañana?, ¿qué tan probable es que un inventario en una empresa aumente a fin de mes?, ¿cuál es la probabilidad de que dos compañías se fusionen? (más detalles ver Walpole/Myers 2007, pág. 726).

El bayesianismo empieza a ser ampliamente aceptado por los investigadores y científicos sólo desde hace dos décadas, cuando avanzan las técnicas computacionales para la estimación de parámetros desde esta metodología y desde entonces se viene aplicando en ingeniería, agricultura, ciencia biomédica y ecología, entre otras (Walpole/Myers 2007, pág. 726).

- Dado que en los métodos clásicos de estimación, se interpretan esencialmente las probabilidades como frecuencias relativas,

22 Este nombre de probabilidad subjetiva se presta para ataques contra el bayesianismo, porque dos investigadores pueden partir de probabilidades distintas respecto a un mismo fenómeno; pero se ha demostrado que aún siendo así, llegan a resultados finales muy similares. Lo de subjetivo indica simplemente que hay probabilidades que no encajan dentro del enfoque frecuentista, pero son estimadas racionalmente por los investigadores del fenómeno en cuestión. 
por ejemplo, al obtener un intervalo de confianza del 95\% para la media poblacional $\mu$, se interpreta como que un $95 \%$ de las veces en experimentos repetidos $Z$ caerá entre -1.96 y 1.96 .

- En el pensamiento bayesiano, a diferencia del enfoque clásico o frecuentista, se parte de distribuciones de probabilidad a priori, y a la luz de evidencia empírica utilizable, se llega a distribuciones de probabilidad posteriores (Wisniewski 2002, pág. 80). Es decir, que en el enfoque bayesiano se tiene muy en cuenta el conocimiento acumulado del investigador respecto a un problema, lo cual no ocurre en el frecuentismo, donde sólo se confía en los resultados obtenidos en el experimento.

- Las pruebas de hipótesis frecuentistas sólo admiten dos resultados: se rechaza la hipótesis a la luz de los resultados obtenidos en la muestra o se reserva el juicio ${ }^{23}$. El enfoque bayesiano presenta una gama más amplia de opciones, porque permite calcular la probabilidad de que una hipótesis sea verdadera, lo que no ocurre en el enfoque clásico, donde sólo tiene sentido hablar de la probabilidad de un suceso mas no de una hipótesis (Díaz 2007, pág. 41).

- La diferencia fundamental entre los enfoques bayesiano y clásico o frecuentista, está en que en los conceptos bayesianos los parámetros se consideran variables aleatorias (media poblacional $\mu$, varianza poblacional $\sigma^{2}$ etc., etc.). Walpole/Myers 2007, pág. 725). En el frecuentismo, los estadísticos (media muestral $\bar{X}$, varianza muestral $S^{2}$...etc.) siguen distribuciones de probabilidad como la normal, $J i$-cuadrado o $t$ student, pero los parámetros se consideran fijos.

Aunque como se verá al final, el enfoque frecuentista ha mostrado grandes aciertos y virtudes a nivel pragmático, también presenta falencias que invitan a los investigadores a tener en

23 En este sentido los test de hipótesis de Fisher, Neyman y Pearson, tienen afinidades con la idea popperiana de buscar en las teorías la refutación y no la aceptación. Una hipótesis estadística nunca se acepta como cierta (más bien se reserva el juicio) y se busca probar su falsedad. 
cuenta otros enfoques como el bayesiano. A continuación se discuten brevemente los cuestionamientos clásicos al frecuentismo.

\subsection{Cuestionamientos al enfoque frecuentista}

(Silva y Muñoz 2000) ${ }^{24}$ adelantan un interesante debate sobre métodos frecuentistas vs bayesianos, que develan las principales críticas a la estadística clásica:

- Ronald Fisher, en la década del 20 del siglo pasado, propuso por primera vez un método para poner a prueba una hipótesis $H_{0}$ mediante una observación empírica $d_{0}$ e introdujo el famoso $p$-value o

$$
P=\operatorname{Probabilidad}\left(D>D_{0} \mid H_{0} \text { cierta }\right)
$$

Es decir, $\mathrm{P}$ es la probabilidad de observar algo mayor a lo que objetivamente se observó suponiendo que $H_{0}$ es cierta.

La principal crítica a este procedimiento radica en que se puede obtener la misma $P$, tanto ante una diferencia observada muy grande si $n$ es pequeño, como ante una diferencia pequeña si $n$ es muy grande (2000, pág. 483). Savage argumenta que cuando se sabe con anterioridad que $H_{0}$ es falsa, el rechazo o la aceptación sólo depende del tamaño de la muestra $n$ y por lo tanto las pruebas no contribuyen a la ciencia. Es decir, que vamos a tomar una decisión en función de cuán pequeño sea el valor de $P$, pero podemos conseguir que $P$ sea tan pequeño como queramos (2000, pág. 483).

24 En la XVII reunión científica SEE celebrada en Santiago de Compostela entre 27-29 de octubre de 1999, Luis Carlos Silva hace de bayesiano (sin serlo abiertamente) y Álvaro Muñoz de frecuentista. Por ser dos autoridades reconocidas en el campo de la investigación estadística, el debate vislumbra grandes posibilidades para la aplicación de los métodos bayesianos, sobre todo por la proliferación de escritos científicos relacionados con este enfoque y las posibilidades de usar herramientas computacionales, que no se tenía en décadas anteriores. 
"Desde un punto de vista lógico-metodológico destacan dos aspectos del test de significación: primero, que siempre que la medida $D$ de desviación exceda el límite de significación $D_{0}$, habrá que considerar la hipótesis como experimentalmente refutada; lo que se apresura a señalar Cramer, no equivale a una refutación lógica de la misma, pues incluso si la hipótesis fuese verdadera, el acontecimiento $D>D_{0}$ de probabilidad $\varepsilon$ podría ocurrir excepcionalmente. En todo caso, cuando $\varepsilon$ es muy pequeño nos consideraremos prácticamente justificados a despreciar esta posibilidad. Así pues, $\varepsilon$ representa la probabilidad de rechazar erróneamente la hipótesis considerada en el caso que sea verdadera; y dado que $\varepsilon$ puede ser elegido arbitrariamente, es posible reducir tal probabilidad de error a un valor convenido" (Rivadulla 1991, pág. 153).

Neyman y Pearson perfecciona los test de hipótesis introduciendo la hipótesis alternativa y las regiones de rechazo y aceptación, pero la elección del nivel de significación sigue siendo arbitraria, lo mismo que el nivel de confianza para la estimación por intervalos ${ }^{25}$. (Rivadulla 1991, pág, 166, 151).

Muchos estadísticos consideran buena la práctica de seleccionar un nivel de significación $\alpha$ antes de realizar la prueba de hipótesis. Este es un procedimiento particularmente bueno cuando se realiza el método del valor $P$, ya que podemos vernos tentados a ajustar el valor del nivel de significación con base en los resultados obtenidos (Triola 2004, pág. 378).

Por ejemplo, con $\alpha=0.05$ y un valor $P=0.06$, no se debería rechazar la hipótesis testada, pero es tentador decir que 0.06 es pequeña

25 Suponga que se va a poner a prueba la hipótesis de que la puntuación promedio en un curso es $\mu=6.45\left(H_{0}\right)$. Se extrae una muestra aleatoria de 25 estudiantes del curso y se obtiene una puntuación media $\bar{X}=7$ con desviación típica $S=1$.3. Usando la distribución $t$ student se tiene: $t=\frac{(7-6.45)}{\frac{1.3}{\sqrt{25}}} 2.115$; para un nivel de significación $\alpha=1 \%$ la hipótesis se rechaza porque: $2.115<2.797$ (Rivadulla 1991, pág. 155). 
para rechazar dicha hipótesis. Otros estadísticos argumentan que la elección previa de $\alpha$ reduce la utilidad de los valores $P$.

Una de las críticas más fuertes de los frecuentistas al bayesianismo es el uso de probabilidades a priori, conlleva el peligro, de que dos investigadores partan de visiones diferentes respecto a un mismo fenómeno. El bayesianismo acepta la subjetividad de los aprioris, el frecuentismo los esconde (Silva 2000, pág. 988). Es tal la manipulación que se ejerce en muchos países a los resultados de la investigación de estadística clásica que los entes reguladores han debido intervenir exigiendo que siempre que se divulgue por ejemplo un promedio, también se muestren otros valores igual de importantes: varianza, nivel de confianza, máximo error permitido, etc. Aseveraciones como que el salario promedio en Europa es de 2.000 euros mensuales, sabemos que no tienen ninguna utilidad práctica si no se asocia a este promedio una medida de dispersión ${ }^{26}$ como la varianza. En el bayesianismo la incertidumbre va acompañada con el resultado y es comunicable al público; en el frecuentismo la varianza va con letra menuda y la media con letra grande (Silva 2002, pág. 487).

- Mientras para realizar una investigación sobre un tema específico, en el bayesianismo se requiere un conocimiento sustantivo del problema, el frecuentismo sólo acude a los datos y a los resultados arrojados por un paquete estadístico, lo cual se presta para el abuso por parte de principiantes en la interpretación de resultados de una investigación. En el bayesianismo se requiere de conocimiento para hacer el análisis. Por ejemplo, en el caso de la medicina, para el bayesiano el eje de investigación

26 Tomemos un ejemplo hipotético y un poco exagerado, midiendo el salario semanal en euros de tres empleados de dos empresas distintas $A$ y $B:$ empresa $A(300,400,500)$, empresa $B(900,200,100)$. En ambos casos se obtiene el mismo salario promedio para las dos empresas $\bar{X}=400$, pero aun siendo iguales estos resultados, a nivel práctico sólo es real el resultado para la empresa $A$ (salarios más homogéneos y cercanos al promedio). El promedio de la empresa $B$ es totalmente falso, desorientador y carente de valor pragmático. 
es la epidemiología, para el frecuentista la estadística (Silva 2000, pág. 488).

- Por el hecho de que los frecuentistas hayan tenido éxito en muchas predicciones y argumenten racionalmente la aceptación de niveles de incertidumbre y error asociados a toda investigación, no se puede aplicar esta metodología indiscriminadamente a todo tipo de problemas y se debe albergar la posibilidad de otras miradas y enfoques. Un nivel de significación $\alpha=1 \%$ en las pruebas de hipótesis, se podría interpretar, que si somos consecuentes con esa regla, se rechazará una hipótesis siendo cierta, no más que una de cada cien veces y la rechazaremos con alta frecuencia cuando sea falsa. Esto parece un procedimiento muy confiable y plausible, pero llevado a nivel pragmático, sería similar a un sistema de justicia al cual no le interesa si un individuo en concreto es culpable o inocente y sólo trata de minimizar el número de veredictos incorrectos (Silva 2000, pág 489). A nivel científico, se debe buscar conclusiones acertadas para cada estudio individual, más que tratar de que el número de veces que nos equivoquemos sea bajo.

Con los ejemplos mencionados anteriormente, no se busca cuestionar los procedimientos de la estadística clásica que a nivel pragmático han mostrado gran utilidad ${ }^{27}$, sino que se pretende sustentar la tesis planteada al inicio del capítulo, que ante las limitaciones de la estadística frecuentista, el bayesianismo surge

27 Sólo aquellos ingenuos que creen que lo estadístico sólo se ocupa de encuestas políticas, se atreven a cuestionar las maravillosas virtudes de la inducción probabilística y de la inferencia estadística: ¿No es acaso por inducción probabilista, que las grandes empresas del mundo estiman sus ventas y utilidades futuras o que las empresas aseguradoras calculan el valor óptimo a cobrar por una póliza, sin poner en riesgo su estabilidad económica y con alto nivel de competitividad? ¿No es acaso por inferencia estadística; que un ingeniero estima los niveles futuros de lluvias y caudales, para el diseño óptimo de una represa? Desde que el hombre primitivo tuvo uso de razón, ha usado el pensamiento inferencial (inductivo) para garantizar su supervivencia: las cosechas, los periodos de sequía, las avalanchas o crecientes y muchos otros fenómenos han sido controlados por medio de inferencias. 
como una alternativa para la toma de decisiones, considerando el conocimiento sustantivo del investigador para la solución de un problema específico. A continuación se presentan argumentos a favor de la viabilidad de una estadística bayesiana, sobre todo enfatizando en que sus modelos son tan consistentes como los del frecuentismo y que las críticas se centran en el uso de distribuciones de probabilidad a priori. Nosotros realizamos el análisis desde un punto de vista netamente pragmático y no profundizamos (porque no es el objetivo del artículo) en otros aspectos importantísimos de tipo epistemológico o que profundizan demasiado en el uso de sofisticadas herramientas matemáticas ${ }^{28}$.

\subsection{Viabilidad del uso del modelo bayesiano en la investigación científica}

Si no se considera el cuestionamiento básico al bayesianismo de usar probabilidades a priori, e incluso obviando la discusión acerca de su carácter inductivo, los únicos argumentos que se encuentran razonables para rechazar dicho enfoque, no ponen en momento alguno en tela de juicio el carácter científico de esta metodología: la estadística frecuentista se presta más para regular y lograr el consenso entre los investigadores, existe una mayor cantidad de softwares y literatura relacionada con el frecuentismo o el hecho de que a nivel mundial se implementa la enseñanza más del frecuentismo. Todos estos argumentos se podrían reunir en uno solo: "La aceptación de cualquier nuevo paradigma tiene más detractores que defensores", no es fácil aceptar que hay una mejor forma de hacer todo lo que hemos hecho hasta ahora". A continuación se presentan argumentos, que si bien es cierto no

28 Luc Bovens y Stephan Hartmann, en bayesian epistemology, realizan un profundo análisis de este enfoque y problemas asociados a él: bayesian coherentism (pág. 10 a 55), reliability (pág. 56 a 88), confirmation (pág. 89 a 111). Clark Glymour (1996) en why I are not bayesian, también aborda las teorías de confirmación y de evidencia. Rivadulla (1991), realiza una espléndida conexión entre la inducción, la deducción y las estadísticas frecuentista y bayesiana. 
colocan al bayesianismo por encima del frecuentismo, al menos lo ubican al mismo nivel:

- (Canavos George 1999, pág. 275, 289) muestra un mismo problema de estimación por intervalos de confianza, resuelto primero por estadística clásica y posteriormente por el enfoque bayesiano ${ }^{29}$ :

TABLA 1. INTERVALOS OBTENIDOS POR CADA ENFOQUE

\begin{tabular}{|c|c|c|}
\hline \multicolumn{3}{|c|}{ ENFOQUE FRECUENTISTA } \\
\hline Confianza & $\begin{array}{c}\text { Limite } \\
\text { inferior }\end{array}$ & $\begin{array}{c}\text { Limite } \\
\text { superior }\end{array}$ \\
\hline $90 \%$ & 501.69 & 505.81 \\
\hline $95 \%$ & 501.30 & 506.20 \\
\hline $99 \%$ & 500.53 & 506.97 \\
\hline \multicolumn{3}{|c|}{ ENFOQUE BAYESIANO } \\
\hline Probabilidad & $\begin{array}{c}\text { Limite } \\
\text { inferior }\end{array}$ & $\begin{array}{c}\text { Limite } \\
\text { superior }\end{array}$ \\
\hline $90 \%$ & 500.18 & 502.75 \\
\hline $95 \%$ & 499.93 & 502.99 \\
\hline $99 \%$ & 499.45 & 503.47 \\
\hline
\end{tabular}

- La estadística bayesiana en los últimos años, dada su gran flexibilidad, ha encontrado en los ensayos clínicos su campo de aplicación más fecundo: "En la estadística frecuentista un diseño dado debe implementarse tal como se previó, es decir, que no puede por ejemplo, aumentarse el tamaño de la muestra

29 En el enfoque clásico, cuando se conoce la varianza, se calcula el intervalo de confianza para la media poblacional $(\mu)$ con la expresión $\bar{X} \pm Z \frac{\sigma}{\sqrt{n}}$.

$\bar{X}$ es la media de la muestra, $\sigma$ la desviación estándar poblacional, $n$ tamaño de la muestra y $Z$ depende del nivel de confianza: por ejemplo $Z=1.645$ para un nivel de confianza del $90 \%$. 
en medio de una experiencia si los datos que arrojó un primer intento experimental no resultaron suficientemente elocuentes. Dentro del marco bayesiano, se continúa la actualización de los criterios que tengamos siempre que haya nuevos datos y el tamaño muestral no ha de establecerse antes. Así, un experimento puede detenerse en cualquier momento y por cualquier razón. El análisis no depende de la estructura del diseño, ni de lo que se haya planificado de antemano" (Silva 2000, pág. 482).

- En los últimos años han aumentado considerablemente las publicaciones relacionadas con el enfoque bayesiano ${ }^{30}$ y los softwares de orientación bayesiana (Silva 200, pág. 76): PAC para diseños experimentales y métodos de comparación de medias y varianzas. WinBugs que permite cálculos complejos bayesianas basados en simulación. Bacc con énfasis en la combinación de modelos y desarrollo de distribuciones predictivas. Hay algunas direcciones de Internet donde se amplía la información sobre softwares bayesianos ${ }^{31}$.

Aunque es considerablemente más común la enseñanza de la estadística frecuentista a nivel de pregrado, ya se empiezan a implementar propuestas tendientes a estimular la enseñanza de los métodos bayesianos. (Díaz, 2007) en su tesis doctoral de la universidad de Granada, donde se defiende la viabilidad de la enseñanza de la inferencia bayesiana en el área de la psicología.

30 Para ilustrar el auge del bayesianismo en la medicina (Silva 2002, pág. 482) busca las citas en Medline a partir de 1966 en títulos y resúmenes de artículos: en la primera década revisada bayesian se citó en 29 títulos y en ningún resumen. Entre 1995 y 1999 se citó en 235 títulos y en 577 resúmenes. También es muy relevante el hecho que autores reconocidos como Freedman o Goodman se hayan interesado por el tema.

31 http://www2.cirano.9c.ca/ bacc/index.html1,http://www.mas.ncl.ac.uk/ ndjw/ bookmarks/stats/software-statistical_computing/bayesian_software/, http://www. mrcbsu.cam.ac.uk/bugs/, http://www.shef.ac.uk/ atyao/1b.html. 
- Los modelos bayesianos se obtienen con una coherencia y rigurosidad matemática, tan confiable como ocurre con la estadística clásica o frecuentista. Se sabe que el mismo teorema de Bayes (el llamado viejo teorema de Bayes) se obtiene deductivamente a partir de los axiomas de probabilidad. La versión moderna del teorema, en áreas como la epidemiología, trabaja con índices de sensibilidad, especificidad y valores predictivos.

Un ejemplo sencillo mostrado en Doval 2004, pág. 7, muestra realmente cómo funciona la vieja versión del teorema de bayes, el cual no admite objeciones ni en su formulación matemática ni en el cálculo de las probabilidades a priori, porque éstas son el resultado de estudios serios y confiables. Las objeciones resultan en la versión moderna de este teorema, que se explica brevemente a continuación contextualizado en la medicina y específicamente en las pruebas diagnósticas.

Al igual que el enfoque frecuentista, el bayesiano no escapa a las críticas y tiene limitaciones y umbrales que demarcan su utilidad a nivel pragmático ${ }^{32}$ relacionados con el problema específico que se quiere resolver. De todas formas, es un enfoque muy prometedor en el campo de la medicina y concretamente en las pruebas diagnósticas. (Escrig 2006) contextualiza los conceptos abordados en este capítulo a problemas reales y rutinarios de la medicina, como es decidir si hay que aplicar determinada prueba o aplicar cierto tratamiento.

\section{Conclusiones}

Se ha tratado de brindar argumentos de tipo pragmático respecto a tres cuestionamientos básicos que se podrían reunir en uno solo: ¿Es posible aprender inductivamente de la experiencia?

32 (Escrig-sos 2006, pág. 272, 273) hace un importante análisis acerca de los intervalos en los cuales las pruebas diagnósticas son o no útiles. (Doval 2004, 9, 10, 11) realiza un análisis completo de un caso real, para mostrar que no siempre las pruebas diagnósticas son útiles y que hay ocasiones en que no brindan ninguna información. 
Ante las dificultades de la inducción lógica para responder satisfactoriamente a esta pregunta, se han buscado en la inducción probabilista y específicamente en la inferencia estadística, los argumentos favorables a nuestra posición de que sí es posible aprender inductivamente de la experiencia.

Como son tangibles las bondades de la estadística general, se ha tratado de mostrar que estos dos enfoques no sólo permiten aprender de la experiencia, sino que admiten la combinación inducción/deducción en su desarrollo y aplicación. Con esto, se considera que se han proporcionado argumentos determinantes para defender nuestra percepción personal, siendo muy conscientes de los alcances o limitaciones de ambos enfoques estadísticos en los que hay que tener muy en cuenta el contexto y el universo reducido en el cual tienen validez los modelos.

Refiriéndose a las aplicaciones bayesianas en medicina, Escrig señala "Cualquier prueba diagnóstica tiene su espacio vital donde es útil; por debajo su aplicación es innecesaria, y por encima puede estar distorsionada en cuanto a su competencia” (Escrig 2006, pág. 273). Respecto al comentario anterior, los resultados obtenidos de una investigación no pueden ser extrapolables de un contexto a otro, dependen mucho de la prevalencia de la enfermedad que haya en un ambiente concreto o población blanca bien definida, aun considerando constantes la sensibilidad y la especifidad (Escrig 2006, pág. 270).

Sin estar en contra de las técnicas frecuentistas, se trata de justificar cómo el bayesianismo es promisorio como una alternativa más adecuada en situaciones donde la estadística clásica presenta problemas. (Bacallao 1996, pág. 467) sugiere que en las investigaciones biomédicas, los esquemas clásicos basados en las pruebas de significación (Fisher) y en la verificación de hipótesis (NeymanPearson) resultan, sino inapropiados, por lo menos insuficientes para afrontar una gran cantidad de problemas muy frecuentes, especialmente en la investigación clínica y epidemiológica. 


\section{Biblografía}

1. Walpole/Sharon. Ronald E. Myers L. Probabilidad y estadística. Pearson. Prentice Hall. México 2007.

2. Triola Mario F. Probabilidad y estadística. Novena edición. Editorial Pearson Educación. México 2004.

3. Aisa Moreu Diego El razonamiento inductivo en la ciencia y en la prueba judicial. Prensas Universitarias de Zaragoza 1977.

4. Rivadulla Andrés. Probabilidad e inferencia científica. Barcelona 1991. Editorial Anthropos.

5. Vickers John. The problem of induction. 2006. Stanford Encyclopedia of philosophy.

6. Norton Jhon D. A material theory of induction. 2003. Universidad de Pittsburg.

7. Norton Jhon D. Induction without probabilities. 2006. Universidad de Pittsburg.

8. Saatsi Juha T. Material theory of induction and scientific realism. 2007. Universidad de Pittsburg.

9. Silva Luis C. Muñoz Álvaro. Debate sobre métodos Frecuentistas VS Bayesianos. Gaceta sanitaria 2000; 14(6).

10. Escrig-sos Javier, Martínez David, Miralles Juan M. Pruebas Diagnósticas: Nociones básicas para su correcta interpretación y uso. Cirugía Española Vol. 79 número 05 del 2006. 
